\title{
INVERSE SCATTERING PROBLEM FOR STURM-LIOUVILLE OPERATOR ON ONE-VERTEX NONCOMPACT GRAPH WITH A CYCLE
}

\author{
MIKHAIL IGNATYEV
}

\begin{abstract}
A scattering problem is studied for second-order differential operator on onevertex noncompact graph with a cycle and with standard matching conditions in the vertex. A uniqueness theorem for a corresponding inverse problem is proved and a procedure for solving the problem is provided.
\end{abstract}

\section{Introduction}

Transport, spectral and scattering problems for differential operators on graphs often appear in mathematics, natural sciences and engineering [1], [3], [4], [2], [5], [6]. During the last years such problems were in the focus of intensive investigations. The most complete results on (both direct and inverse) spectral problems were achieved in the case of compact graphs [8], [7], [9], [10], [11], [12], [13]. In the noncompact case there are no similar general results since the presence of the noncompact edges (rays) leads to new qualitative difficulties in the investigation of the spectral problems. Some particular results in this direction were obtained in [14], [15], [16], [17].

The present paper is devoted to the Sturm-Liouville operator on a one-vertex geometric graph which consists of one cycle (or: closed walk) and a finite number of rays emanating from the vertex. The paper is structured as follows. In section 2 we establish some spectral properties of the considered operator. Section 3 is devoted to a certain particular inverse scattering problem that consists in recovering the potential on one ray from the given part of scattering data. In section 4 we provide a formulation of the inverse scattering problem and give a constructive procedure for solving this problem.

Let $v$ be some point in a finite-dimensional Euclidian space. Let $\mathscr{E}_{0}$ be a smooth curve of length $\pi$ which starts and ends in $v$, further let $\mathscr{E}_{j}, j=\overline{1, p}$ be rays emanating from $v$. We assume $p>1$, case $p=1$ requires special consideration. Suppose that each ray does not

2000 Mathematics Subject Classification. 34A55, 34B24, 47E05.

Key words and phrases. Sturm-Liouville operators, noncompact graph, graph with a cycle, scattering problems, inverse spectral problems. 
intersect any other ray and does not intersect $\mathscr{E}_{0}$. Define $G:=\bigcup_{j=0}^{p} \mathscr{E}_{j}$. We consider $G$ as a geometric graph with the vertex $v$ and the set of edges $\mathscr{E}=\left\{\mathscr{E}_{j}\right\}, j=\overline{0, p}$. We assume that all the edges are parameterized with the natural parameters $x_{j}, x_{j} \in[0, \infty), j=\overline{1, p}, x_{0} \in[0, \pi]$.

The function $y(x)$ on $G$ we shall treat as a vector $y(x)=\left[y_{j}\left(x_{j}\right)\right]_{j=0}^{p}$. Consider the differential expressions:

$$
\ell_{j} y_{j}:=-y_{j}^{\prime \prime}+q_{j}\left(x_{j}\right) y_{j}
$$

where the potential function $q(x)$ is real-valued and satisfying the following condition

$$
\int_{0}^{\pi}\left|q_{0}\left(x_{0}\right)\right| d x_{0}+\sum_{j=1}^{p} \int_{0}^{\infty}\left(1+x_{j}\right)\left|q_{j}\left(x_{j}\right)\right| d x_{j}<\infty .
$$

The following condition in a vertex $v$ we call standard matching condition:

$$
\sum_{j=0}^{p} y_{j}^{\prime}(0)=y_{0}(\pi) .
$$

We consider in $L_{2}(G) \cap C(G)$ (which is considered as a dense subset of $L_{2}(G)$ ) the SturmLiouville operator $L=L(q, G)$ which is generated by the expression (1.1) and the matching condition (1.3).

\section{Auxiliary propositions}

We call a function $\Phi(x, \lambda)$, defined at least for $x \in G, \lambda \in \mathbf{C} \backslash \mathbf{R}$ the Weyl solution iff:

(1) $\Phi(\cdot, \lambda) \in L_{2}(G) \cap C(G)$ and $\left.\Phi\right|_{\nu}=1$;

(2) it solves the differential equations $\ell_{j} \Phi_{j}=\lambda \Phi_{j}, j=\overline{0, p}$.

The value

$$
M(\lambda):=\sum_{j=0}^{p} \Phi_{j}^{\prime}(0, \lambda)-\Phi_{0}^{\prime}(\pi, \lambda)
$$

is called the Weyl function. Although we shall not use $M(\lambda)$ as an input data for the inverse problem it plays a significant role in our further considerations.

We recall that a Nevanlinna function $F$ is by definition defined and holomorphic at least in $\mathbf{C} \backslash \mathbf{R}$ and has the properties

- $F(z)=\overline{F(\bar{z})}$ for all $z$ in the domain of holomorphy of $F$.

- $\frac{\operatorname{Im} F(z)}{\operatorname{Im} z} \geq 0$ for $\operatorname{Im} z \neq 0$. 
Lemma 2.1. $M(\lambda)$ is a Nevanlinna function.

Proof. In order to construct the Weyl solution $\Phi(x, \lambda)$ one can use a local fundamental system of solutions on each edge. Then standard technique [9]-[13] reduces the condition 1 to a certain system of linear algebraic equations with a determinant which is a holomorphic function in $\mathbf{C} \backslash[0,+\infty)$. So $\Phi(x, \lambda)$ exists and is unique for all $\lambda \in \mathbf{C} \backslash[0,+\infty)$ with the possible exception of a countable set of points and moreover the Weyl function $M(\lambda)$ is meromorphic in $\mathbf{C} \backslash[0,+\infty)$. Further one can easily verify that $M(\bar{\lambda})=\overline{M(\lambda)}$.

Now let us take an arbitrary $\lambda=\sigma+i \tau$. Then we can apply the following classical identity (see for instance [18]):

$$
\tau \int_{0}^{T}|y|^{2} d x=\operatorname{Im}\left(\overline{y(0)} y^{\prime}(0)\right)-\operatorname{Im}\left(\overline{y(T)} y^{\prime}(T)\right)
$$

to the Weyl solution on each edge. This yields:

$$
\begin{gathered}
\tau \int_{0}^{\pi}\left|\Phi_{0}\right|^{2} d x_{0}=\operatorname{Im}\left(\overline{\Phi_{0}(0)} \Phi_{0}^{\prime}(0)\right)-\operatorname{Im}\left(\overline{\Phi_{0}(\pi)} \Phi_{0}^{\prime}(\pi)\right), \\
\tau \int_{0}^{\infty}\left|\Phi_{j}\right|^{2} d x_{j}=\operatorname{Im} \overline{\Phi_{j}(0)} \Phi_{j}^{\prime}(0) \quad j=\overline{1, p}
\end{gathered}
$$

Summarizing these identities and using the condition 1 we obtain:

$$
\tau \int_{0}^{\pi}\left|\Phi_{0}\right|^{2} d x_{0}+\tau \sum_{j=1}^{p} \int_{0}^{\infty}\left|\Phi_{j}\right|^{2} d x_{j}=\operatorname{Im} M(\lambda)
$$

and we can conclude that $\operatorname{Im} M(\lambda)$ and $\operatorname{Im} \lambda$ have the same sign.

Let $C_{j}\left(x_{j}, \lambda\right), S_{j}\left(x_{j}, \lambda\right), j=\overline{0, p}$ be the solutions of the equations $\ell_{j} y_{j}=\lambda y_{j}$ under the initial conditions $C_{j}(0, \lambda)=S_{j}^{\prime}(0, \lambda)=1, S_{j}(0, \lambda)=C_{j}^{\prime}(0, \lambda)=0$. Also we shall use the Jost solutions $e_{j}\left(x_{j}, \rho\right), j=\overline{1, p}$ of the equations $\ell_{j} y_{j}=\rho^{2} y_{j}$ with the asymptotics $e_{j}\left(x_{j}, \rho\right)=$ $\exp \left(i \rho x_{j}\right)(1+o(1))$ as $x_{j} \rightarrow \infty$.

Now we consider the eigenvalue problem for $L$. In order to obtain negative eigenvalues one can proceed as follows. Let $\lambda \in \mathbf{C} \backslash[0,+\infty)$. Then an eigenfunction has the form:

$$
\begin{aligned}
& y_{j}\left(x_{j}\right)=\gamma_{j} e_{j}\left(x_{j}, \rho\right), j=\overline{1, p}, \\
& y_{0}\left(x_{0}\right)=\alpha_{0} C_{0}\left(x_{0}, \lambda\right)+\beta_{0} S_{0}\left(x_{0}, \lambda\right),
\end{aligned}
$$

where $\lambda=\rho^{2}, \rho \in \Omega_{+}:=\{\rho: \operatorname{Im} \rho>0\}$. In view of (2.1), (2.2) the matching condition (1.3) together with the condition $y(\cdot) \in C(G)$ reduce to a system of linear algebraic equations with respect to the values $\left\{\alpha_{0}, \beta_{0}\right\}$ and $\left\{\gamma_{j}\right\}_{j=\overline{1, p}}$. We denote the determinant of this system $\Delta(\lambda)$ and call it characteristic function for $L$. Clear that for given $\lambda \in \mathbf{C} \backslash[0,+\infty)$ to be an eigenvalue for $L$ it is necessary and sufficient to be a zero of $\Delta(\cdot)$. 
The following lemma can be easily obtained via direct calculation.

Lemma 2.2. The following representation holds:

$$
M(\lambda)=\frac{\Delta(\lambda)}{d(\lambda)}
$$

where

$$
d(\lambda):=\prod_{j=0}^{p} d_{j}(\lambda), d_{j}(\lambda):=e_{j}(0, \rho), d_{0}(\lambda):=S_{0}(\pi, \lambda),
$$

i.e. $d_{j}(\lambda)$ are the characteristic functions for Dirichlet-Dirichlet eigenvalue problems on $\mathscr{E}_{j}$.

Denote $\Lambda$ the set of eigenvalues of $L$. Since $L$ is self-adjoint all the eigenvalues are real. We split $\Lambda$ as follows: $\Lambda=\Lambda^{-} \cup \Lambda^{+}$, where $\Lambda^{-}=\Lambda \cap(-\infty, 0)$. Let $n_{-}=\operatorname{card}\left(\Lambda^{-}\right)$and let $N_{-}$be a number of the negative zeros of $\Delta(\cdot)$ counted with multiplicity.

Lemma 2.3. The following estimate holds:

$$
n_{-} \leq N_{-} \leq N_{0}+Q
$$

where

$$
Q=\sum_{j=1}^{p} \int_{0}^{\infty} x_{j}\left|q_{j}\left(x_{j}\right)\right| d x_{j}
$$

and $N_{0}$ depends only upon $q_{0}(\cdot)$.

Proof. It follows from Lemma 2.2 that the negative zeros of $\Delta(\cdot)$ are interlaced with the negative zeros of $d(\cdot)$. It is known that the number of negative zeros of $d_{0}(\cdot)$ is finite while the number of zeros of $d_{j}(\cdot)$ can be estimated with the value [18]:

$$
Q_{j}=\int_{0}^{\infty} x_{j}\left|q_{j}\left(x_{j}\right)\right| d x_{j} .
$$

Let us introduce the graphs $G^{k}, k=\overline{0, p}$ with the single vertex $v$ and the sets of edges $\left\{\mathscr{E}_{j}\right\}_{j=\overline{0, p} \backslash\{k\}}$. Denote $\Delta^{k}(\cdot)$ the characteristic function for $L$ on $G^{k}$ and $M_{k}(\cdot)$ the corresponding Weyl function. Lemma 2.2 yields the representation:

$$
M_{k}(\lambda)=\frac{\Delta^{k}(\lambda)}{\Delta_{k}(\lambda)}
$$

where

$$
\Delta_{k}(\lambda):=\prod_{j=\overline{0, p} \backslash\{k\}} d_{j}(\lambda) .
$$

Also we introduce the functions:

$$
d^{k}(\lambda)=e_{k}^{\prime}(0, \rho), k=\overline{1, p}, \quad d^{0}(\lambda)=2-C_{0}(\pi, \lambda)-S_{0}^{\prime}(\pi, \lambda) .
$$


Note that the following fractions:

$$
m_{k}(\lambda):=\frac{d^{k}(\lambda)}{d_{k}(\lambda)}
$$

can be treated as "local Weyl functions" on $\mathscr{E}_{k}$ and, in particular, all of them are the Nevanlinna functions.

Direct calculations yield the following results.

Lemma 2.4. The following representation holds:

$$
\Delta(\lambda)=d_{k}(\lambda) \Delta^{k}(\lambda)+d^{k}(\lambda) \Delta_{k}(\lambda)
$$

where $k \in \overline{0, p}$ is arbitrary.

Remark 2.1. It is often convenient to use both spectral parameters $\lambda$ and $\rho$ in the same formula like it has been done in Lemma 2.3. Here and everywhere below we assume $\lambda=\rho^{2}$ and if $\rho \in \mathbf{R} \backslash\{0\}$ we agree that $\lambda=\rho^{2}+\operatorname{sgn} \rho \cdot i 0$ (i.e. on the boundary of the cut in $\mathbf{C} \backslash[0,+\infty)$ one should take here and below the corresponding limit).

Lemma 2.5. For a.e. $\lambda \in(0, \infty)$ one has $\pm \operatorname{Im} M_{k}(\lambda \pm i 0)>0$. If $k=0$ then the estimate holds for all $\lambda \in(0, \infty)$.

Proof. The assertion follows directly from the representation:

$$
M(\lambda)=\sum_{j=0}^{p} m_{j}(\lambda)
$$

and the analogous representations for $M_{k}(\lambda)$. In addition we recall that [19]

$$
\pm \operatorname{Im} m_{j}(\lambda \pm i 0)>0, \lambda \in(0, \infty), j=\overline{1, p} .
$$

Lemma 2.6. The following estimates hold:

$$
\begin{aligned}
& |\Delta(\lambda)| \geq\left|\Delta_{k}(\lambda)\right| \cdot\left|d_{k}(\lambda)\right| \cdot\left|\operatorname{Im} m_{k}(\lambda)\right|, k=\overline{1, p}, \\
& |\Delta(\lambda)| \geq\left|\Delta^{k}(\lambda)\right| \cdot\left|d^{k}(\lambda)\right| \cdot\left|\operatorname{Im} \frac{1}{m_{k}(\lambda)}\right|, k=\overline{1, p} ; \\
& |\Delta(\lambda)| \geq\left|\Delta_{0}(\lambda)\right| \cdot\left|d_{0}(\lambda)\right| \cdot\left|\operatorname{Im} M_{0}(\lambda)\right| \\
& |\Delta(\lambda)| \geq\left|\Delta^{0}(\lambda)\right| \cdot\left|d^{0}(\lambda)\right| \cdot\left|\operatorname{Im} \frac{1}{M_{0}(\lambda)}\right|,
\end{aligned}
$$

where $\lambda=\rho^{2}, \rho \in \overline{\Omega_{+}} \backslash\{0\}$.

Proof. Let $\lambda$ be such that $\Delta_{0}(\lambda) d_{0}(\lambda) \neq 0$. Then Lemma 2.4 in view of Lemma 2.2 yields:

$$
\frac{\Delta(\lambda)}{\Delta_{0}(\lambda) d_{0}(\lambda)}=M_{0}(\lambda)+m_{0}(\lambda)
$$


Since both $M_{0}(\cdot)$ and $m_{0}(\cdot)$ are Nevanlinna functions the values $\operatorname{Im} M_{0}(\lambda)$ and $\operatorname{Im} m_{0}(\lambda)$ have the same sign. So we have:

$$
\left|M_{0}(\lambda)+m_{0}(\lambda)\right| \geq\left|\operatorname{Im} M_{0}(\lambda)+\operatorname{Im} m_{0}(\lambda)\right| \geq\left|\operatorname{Im} M_{0}(\lambda)\right|
$$

Another estimates can be obtained analogously.

Lemma 2.7. If $\lambda \in(0,+\infty)$ is such that $\Delta(\lambda)=0$ then $\lambda \in \Lambda$. Conversely if $\lambda \in \Lambda^{+}$then $\Delta(\lambda)=0$.

Remark 2.2. Generally, for $\lambda \in(0,+\infty)$ we have two different values $\Delta(\lambda \pm i 0)$ (see also Remark 2.1). Nevertheless, from the relation $\Delta(\bar{\lambda})=\overline{\Delta(\lambda)}$ it follows that $\Delta(\lambda+i 0)=0$ implies $\Delta(\lambda-i 0)=$ 0 and vice versa. So in Lemma 2.7 and its proof below the notation $\Delta(\lambda)$ would not effect any confusion.

Proof of Lemma 2.7. It follows from Lemma 2.6 $(k=0)$ that $\Delta(\lambda)=0$ for real positive $\lambda$ is possible only if $d_{0}(\lambda)=d^{0}(\lambda)=0$. This means that $\lambda$ is an eigenvalue for both Dirichlet-Dirichlet and periodic problems on $\mathscr{E}_{0}$. In this situation $S_{0}\left(x_{0}, \lambda\right)$ satisfies the periodic boundary conditions and the following function:

$$
y(x)=\left\{\begin{array}{l}
0, x \in \mathscr{E}_{j}, j=\overline{1, p}, \\
S_{0}\left(x_{0}, \lambda\right)
\end{array}\right.
$$

is an eigenfunction for $L$ on entire $G$.

Conversely, if real non-negative $\lambda$ is an eigenvalue for $L$ on $G$ then the corresponding eigenfunction must be identically 0 on each of $\mathscr{E}_{j}, j=\overline{1, p}$. Consequently, on $\mathscr{E}_{0}$ it must be proportional to $S_{0}\left(x_{0}, \lambda\right)$ and the matching conditions (1.3) reduce to the periodic conditions on $S_{0}\left(x_{0}, \lambda\right)$. This means that $\lambda$ is an eigenvalue for both Dirichlet-Dirichlet and periodic problems on $\mathscr{E}_{0}, d_{0}(\lambda)=d^{0}(\lambda)=0$.

Lemma 2.8. For $\lambda=\rho^{2},|\rho|>\rho^{*}, \rho \in \overline{\Omega_{+}^{\delta}}=\left\{\rho \in \overline{\Omega_{+}}:|\rho-n| \geq \delta, n \in \mathbf{Z}\right\}$ the following estimate holds:

$$
C_{\delta} \exp (\tau \pi) \leq|\Delta(\lambda)| \leq C \exp (\tau \pi)
$$

where $\tau=\operatorname{Im} \rho$.

Furthermore,

$$
\Delta(\lambda)=-\frac{p+2}{2} \exp (\tau \pi)+O\left(\frac{1}{\rho} \exp (\tau \pi)\right), \rho \rightarrow \infty, \arg \rho \in[\varepsilon, \pi-\varepsilon], \varepsilon \in(0, \pi / 2) .
$$

Proof. Using Lemma 2.4 one can obtain the following representation:

$$
\Delta(\lambda)=\sum_{k=0}^{p} d^{k}(\lambda) \prod_{j=\overline{1, p} \backslash\{k\}} d_{k}(\lambda) .
$$


Using here the classical asymptotics:

$$
d_{k}(\lambda)=1+O\left(\frac{1}{\rho}\right), d^{k}(\lambda)=i \rho\left(1+O\left(\frac{1}{\rho}\right)\right), k=\overline{1, p}
$$

and

$$
d_{0}(\lambda)=\frac{\sin \rho \pi}{\rho}+O\left(\frac{1}{\rho^{2}} \exp (\tau \pi)\right), d^{0}(\lambda)=2-2 \cos (\rho \pi)+O\left(\frac{1}{\rho} \exp (\tau \pi)\right)
$$

we arrive at:

$$
\Delta(\lambda)=2-2 \cos (\rho \pi)+i p \sin \rho \pi+O\left(\frac{1}{\rho} \exp (\tau \pi)\right) .
$$

Now standard arguments [18], [19] complete the proof.

\section{Particular scattering problem}

Let us take an arbitrary ray $\mathscr{E}_{k}, k \in \overline{1, p}$. We call the function $\psi_{k}(x, \rho), x \in G, \rho \in \Omega_{+}$the Weyl-type solution associated with $\mathscr{R}_{k}$ iff:

(1) it is continuous on $G$ (with respect to $x$ ) and satisfying (1.3);

(2) it solves the differential equation $\ell_{j} \psi_{k j}=\rho^{2} \psi_{k j}, j=\overline{0, p}$;

(3) $\psi_{k j}\left(x_{j}, \rho\right)=O\left(\exp \left(i \rho x_{j}\right)\right)$ as $x_{j} \rightarrow \infty, j=\overline{1, p} \backslash\{k\}$;

(4) $\psi_{k k}\left(x_{k}, \rho\right)=\exp \left(-i \rho x_{k}\right)(1+o(1))$ as $x_{k} \rightarrow \infty$.

In order to construct $\psi_{k}(x, \rho)$ one can use the following representations:

$$
\begin{array}{r}
\psi_{k j}\left(x_{j}, \rho\right)=\gamma_{k j}(\rho) e_{j}\left(x_{j}, \rho\right), \quad j=\overline{1, p} \backslash\{k\} ; \\
\psi_{k k}\left(x_{k}, \rho\right)=\gamma_{k k}(\rho) e_{k}\left(x_{k}, \rho\right)+\delta_{k}(\rho) S_{k}\left(x_{k}, \lambda\right) .
\end{array}
$$

Clear that these representations guarantee that conditions (2) - (3) are satisfied. Then (4) is equivalent to

$$
\delta_{k}(\rho)=-\frac{2 i \rho}{e_{k}(0, \rho)}
$$

Finally condition 1) leads to a linear algebraic system (with respect to $\left\{\gamma_{k j}\right\}$ ) whose determinant coincides with characteristic function $\Delta(\lambda)$. Solving this system we obtain in particular:

$$
\gamma_{k k}(\rho)=\frac{2 i \rho}{e_{k}(0, \rho)} \cdot \frac{\Delta_{k}(\lambda)}{\Delta(\lambda)},
$$

where $\Delta_{k}(\lambda)$ is the function introduced in previous section.

Also we shall use the following representation.

$$
\gamma_{k k}(\rho)=\frac{2 i \rho}{e_{k}^{2}(0, \rho)} \cdot \frac{1}{M_{k}(\lambda)} .
$$


The considerations above lead us to the following lemma.

Lemma 3.1. For $x_{k} \in[0, \infty) \psi_{k k}\left(x_{k}, \rho\right)$ is a meromorphic function with respect to $\rho$ in $\Omega_{+}$with possible poles on the imaginary axis.

We denote by $Z_{k}^{-}$the set of poles of $\psi_{k k}\left(x_{k}, \rho\right)$. It is clear that .

Lemma 3.2. If $\rho_{0} \in Z_{k}^{-}$then $\lambda_{0}=\rho_{0}^{2} \in \Lambda^{-}$. $Z_{k}^{-}$is a finite set.

Proof. It follows from (3.4), (3.3) that for given $\rho \in \Omega_{+}$to be a pole for $\psi_{k k}\left(x_{k}, \rho\right)$ it is sufficient that $\lambda_{0}=\rho_{0}^{2}$ was a zero of the function $d_{k}(\lambda) \Delta(\lambda)$. Suppose that $d_{k}\left(\lambda_{0}\right)=0$ but $\Delta\left(\lambda_{0}\right) \neq 0$. It is clear that $\psi_{k k}\left(x_{k}, \rho\right)$ has at $\rho=\rho_{0}$ at most a simple pole. Calculating the residue one gets:

$$
\operatorname{res}_{\rho=\rho_{0}} \psi_{k k}\left(x_{k}, \rho\right)=\frac{2 i \rho_{0}}{\dot{e}_{k}\left(0, \rho_{0}\right)} \cdot \frac{\Delta_{k}\left(\lambda_{0}\right)}{\Delta\left(\lambda_{0}\right)} \cdot e_{k}\left(x_{k}, \rho_{0}\right)-\frac{2 i \rho_{0}}{\dot{e}_{k}\left(0, \rho_{0}\right)} \cdot S_{k}\left(x_{k}, \lambda_{0}\right),
$$

where dot denotes a derivative with respect to $\rho$. Now we take into account that in view of Lemma $2.4 \Delta\left(\lambda_{0}\right)=e_{k}^{\prime}\left(0, \rho_{0}\right) \Delta_{k}\left(\lambda_{0}\right)$, while $0=d_{k}\left(\lambda_{0}\right)=e_{k}\left(0, \rho_{0}\right)$ yields $e_{k}\left(x_{k}, \rho_{0}\right)=e_{k}^{\prime}\left(0, \rho_{0}\right) S_{k}\left(x_{k}, \lambda_{0}\right)$ and we obtain finally res $\operatorname{re}_{\rho} \psi_{k k}\left(x_{k}, \rho\right)=0$, i.e. $\rho_{0}$ is not a pole actually. Thus, we have proved that all the poles of $\psi_{k k}\left(x_{k}, \rho\right)$ correspond to the eigenvalues of $L$. Now Lemma 2.3 guarantees that $Z_{k}^{-}$is a finite set.

Lemma 3.3. All poles of $\psi_{k k}\left(x_{k}, \rho\right)$ are simple. For the residue $\operatorname{res}_{\rho=\rho_{0}} \psi_{k k}\left(x_{k}, \rho\right), \rho_{0} \in Z_{k}^{-}$the following asymptotic behavior is valid

$$
\operatorname{res}_{\rho=\rho_{0}} \psi_{k k}\left(x_{k}, \rho\right)=i \alpha_{k}\left(\rho_{0}\right) \exp \left(i \rho_{0} x_{k}\right)(1+o(1)), \quad x_{k} \rightarrow \infty .
$$

The values $\alpha_{k}\left(\rho_{0}\right)$ are all real and positive.

Proof. Let $\rho_{0} \in Z_{k}^{-}$be such that $e_{k}\left(0, \rho_{0}\right) \neq 0$. Then $\lambda_{0}=\rho_{0}^{2}$ is a zero of $\Delta(\lambda)$ and a simple zero of $M_{k}(\lambda)$ from (3.5). Since $M_{k}(\lambda)$ is a Nevanlinna function one has:

$$
\frac{1}{M_{k}(\lambda)}=\frac{a\left(\lambda_{0}\right)}{\lambda-\lambda_{0}}+O(1), \lambda \rightarrow \lambda_{0}
$$

where $a\left(\lambda_{0}\right)$ is real and positive. In view of (3.6) this yields:

$$
\gamma_{k k}(\rho)=\frac{2 i \rho_{0}}{e_{k}^{2}\left(0, \rho_{0}\right)} \cdot \frac{a\left(\lambda_{0}\right)}{\rho^{2}-\rho_{0}^{2}}+O(1), \rho \rightarrow \rho_{0},
$$

and consequently $\gamma_{k k}(\rho)$ has a simple pole in $\rho_{0}$ and

$$
\operatorname{res}_{\rho=\rho_{0}} \gamma_{k k}(\rho)=i \alpha_{k}\left(\rho_{0}\right), \alpha_{k}\left(\rho_{0}\right)=\frac{a\left(\lambda_{0}\right)}{e_{k}^{2}\left(0, \rho_{0}\right)} .
$$

Moreover, since $e_{k}^{2}\left(0, \rho_{0}\right) \in(0,+\infty)$, we have $\alpha_{k}\left(\rho_{0}\right) \in(0,+\infty)$. Now from (3.5), (3.7) we infer that

$$
\operatorname{res}_{\rho=\rho_{0}} \psi_{k k}\left(x_{k}, \rho\right)=i \alpha_{k}\left(\rho_{0}\right) e_{k}\left(x_{k}, \rho_{0}\right) \text {, }
$$


which yields the required asymptotic expression.

Now let $e_{k}\left(0, \rho_{0}\right)=0$. In this case we repeat the arguments above replacing the solution $S_{k}\left(x_{k}, \lambda\right)$ with the solution $S_{k}^{0}\left(x_{k}, \lambda\right)$ under the sine-type conditions at $x_{k}=x_{k}^{0}$, where $e_{k}\left(x_{k}^{0}, \rho\right) \neq 0$.

We call the values $\alpha_{k}\left(\rho_{0}\right), \rho_{0} \in Z_{k}^{-}$the weight numbers.

Remark 3.2. Actually we have proved the following representation:

$$
\operatorname{res}_{\rho=\rho_{0}} \psi_{k k}\left(x_{k}, \rho\right)=i \alpha_{k}\left(\rho_{0}\right) e_{k}\left(x_{k}, \rho_{0}\right), \rho_{0} \in Z_{k}^{-} \text {. }
$$

Now we consider the behavior of $\psi_{k k}\left(x_{k}, \rho\right)$, as $\operatorname{Im} \rho \rightarrow+0$. Denote $Z_{0}^{+}$the set of all $\rho \in \mathbf{R}$ such that $\lambda=\rho^{2} \in \Lambda$.

Lemma 3.4. If $\rho_{0} \in \mathbf{R} \backslash\left(\{0\} \cup Z_{0}^{+}\right)$then there exists the limit $\psi_{k k}\left(x_{k}, \rho_{0}\right):=\lim _{\rho \rightarrow \rho_{0}, \rho \in \Omega_{+}} \psi_{k k}\left(x_{k}, \rho_{0}\right)$. If $\rho_{0} \in Z_{0}^{+}$then $\psi_{k k}\left(x_{k}, \rho\right)$ and $\psi_{k k}^{\prime}\left(x_{k}, \rho\right)$ are bounded as $\rho \rightarrow \rho_{0}, \rho \in \Omega_{+}$.

Proof. The assertion of the lemma follows directly from (3.2)-(3.4) and Lemmas 2.6, 2.4.

Proceeding as in the classical scattering theory we arrive at

Lemma 3.5. For $\psi_{k k}\left(x_{k}, \rho\right), \rho \in \mathbf{R} \backslash\left(\{0\} \cup Z_{0}^{+}\right)$the following representation holds:

$$
\psi_{k k}\left(x_{k}, \rho\right)=e_{k}\left(x_{k},-\rho\right)+s_{k}(\rho) e_{k}\left(x_{k}, \rho\right) \text {. }
$$

Corollary. For $\psi_{k k}\left(x_{k}, \rho\right), \rho \in \mathbf{R} \backslash\left(\{0\} \cup Z_{0}^{+}\right)$the following asymptotics is valid:

$$
\psi_{k k}\left(x_{k}, \rho\right)=\exp \left(-i \rho x_{k}\right)+s_{k}(\rho) \exp \left(i \rho x_{k}\right)+o(1), \quad x_{k} \rightarrow \infty .
$$

We call the function $s_{k}(\rho), \rho \in \mathbf{R} \backslash\left(\{0\} \cup Z_{0}^{+}\right)$the reflection coefficient associated with $\mathscr{E}_{k}$; the corollary shows how it can be measured.

Lemma 3.6. For a.e. $\rho \in \mathbf{R} \backslash\left(\{0\} \cup Z_{0}^{+}\right)\left|s_{k}(\rho)\right|<1$. Furthermore, $s_{k}(-\rho)=\overline{s_{k}(\rho)}$.

Proof. Using (3.2) - (3.4) one can obtain after some algebra:

$$
s_{k}(\rho)=-\frac{e_{k}(0,-\rho)}{e_{k}(0, \rho)} \cdot \frac{M_{k}(\lambda)+\overline{m_{k}(\lambda)}}{M_{k}(\lambda)+m_{k}(\lambda)} .
$$

This representation yields the symmetry $s_{k}(-\rho)=\overline{s_{k}(\rho)}$. Further, it follows from Lemma 2.5 that:

$$
\left|\frac{M_{k}(\lambda)+\overline{m_{k}(\lambda)}}{M_{k}(\lambda)+m_{k}(\lambda)}\right|<1
$$


while the classical theory yields:

$$
\left|\frac{e_{k}(0,-\rho)}{e_{k}(0, \rho)}\right|=1 .
$$

Substituting these estimates into (3.8) we obtain the required assertion.

Lemma 3.7. The following estimate holds:

$$
\left|\gamma_{k k}(\rho)\right| \leq A_{k}, \quad \rho \rightarrow \infty, \rho \in \overline{\Omega_{+}} \backslash\{0\},
$$

where $\gamma_{k k}(\rho)$ is given by (3.4) and $A_{k}$ is a constant depending on the geometry of the graph $G$ and not on $q(\cdot)$.

If in addition $q(\cdot)$ is compactly supported, then the estimate can be made more precise:

$$
\left|g_{k}(\rho)\right| \leq A_{k}, \quad g_{k}(\rho):=\gamma_{k k}(\rho) \prod_{\rho_{0} \in Z_{k}^{-}} \frac{\rho-\rho_{0}}{\rho-\bar{\rho}_{0}}, \rho \in \overline{\Omega_{+}} .
$$

Proof. The first part of the assertion can be obtained in a straightforward way from the representation (3.5), Lemma 2.8.

Now let $q(\cdot)$ be compactly supported. Since for $\rho, \rho_{0} \in \overline{\Omega_{+}}$we have $\left|\rho-\rho_{0}\right| \leq\left|\rho-\overline{\rho_{0}}\right|$ the estimate obtained for $\gamma_{k k}(\rho)$ remains the same for $g_{k}(\rho)$. So we have for all sufficiently large $\rho \in \overline{\Omega_{+}}:\left|g_{k}(\rho)\right| \leq A_{k}$.

Let us consider $\rho \in \mathbf{R} \backslash\{0\}$. One has

$$
s_{k}(\rho)=\gamma_{k k}(\rho)-\frac{e_{k}(0,-\rho)}{e_{k}(0, \rho)}
$$

and we can estimate:

$$
\left|g_{k}(\rho)\right|=\left|\gamma_{k k}(\rho)\right| \leq 2, \rho \in \mathbf{R} \backslash\{0\} .
$$

Since $q(\cdot)$ is compactly supported the function $g_{k}(\rho)$ is holomorphic in a neighborhood of 0 and the estimate (3.9) remains true for all $\rho \in \mathbf{R}$. Now for completing the proof it is sufficient to apply the maximum principal to $g_{k}(\rho)$ in $\Omega_{+} \cap\{\rho:|\rho|<R\}$ with sufficiently large $R>0$.

Remark 3.3. Since for $\rho \in \bar{\Omega}_{+}, \rho_{0} \in \bar{\Omega}_{+}$one has

$$
\left|\frac{\rho-\rho_{0}}{\rho-\bar{\rho}_{0}}\right| \leq 1
$$

the estimate of Lemma 3.7 can be generalized in the following way:

$$
|f(\rho)| \leq A_{k}, \quad f(\rho):=\gamma_{k k}(\rho) \prod_{\rho_{0} \in Z}\left(\frac{\rho-\rho_{0}}{\rho-\bar{\rho}_{0}}\right)^{v\left(\rho_{0}\right)},
$$

where $Z$ is an arbitrary finite subset of $\bar{\Omega}_{+}$such that $Z_{k}^{-} \subseteq Z$ and $v\left(\rho_{0}\right), \rho_{0} \in Z$ are arbitrary nonnegative integer numbers. 
Lemma 3.8. $\psi_{k k}\left(x_{k}, \rho\right), \psi_{k k}^{\prime}\left(x_{k}, \rho\right)$, are bounded as $\rho \rightarrow 0, \rho \in \bar{\Omega}_{+}$.

Proof. If $q(\cdot)$ is compactly supported then the required estimate follows directly from Lemma 3.7. In the general case we consider the family $L^{(T)}=L\left(q^{(T)}(x), G\right)$ with a parameter $T>0$, where:

$$
\begin{aligned}
& q_{0}^{(T)}\left(x_{0}\right)=q_{0}\left(x_{0}\right) ; \\
& q_{j}^{(T)}\left(x_{j}\right)=q_{j}\left(x_{j}\right), x_{j} \leq T ; \\
& q_{j}^{(T)}\left(x_{j}\right)=0, x_{j}>T .
\end{aligned}
$$

Classical Sturm-Liouville theory on the semi-axis shows that $\Delta^{(T)}(\lambda) \rightarrow \Delta(\lambda)$ as $T \rightarrow \infty$ uniformly on $\{\lambda \in \mathbf{C} \backslash[0,+\infty): \varepsilon<|\lambda|<R\}$ with any $0<\varepsilon<R<\infty$. Moreover, if we write $\Lambda^{-}$in the form $\Lambda^{-}=\left\{\lambda_{v}=\rho_{v}^{2}\right\}_{v=\overline{1, n_{-}}}$then it is guaranteed that $\gamma_{k k}^{(T)}(\rho) \rightarrow \gamma_{k k}(\rho)$ as $T \rightarrow+\infty$ uniformly in $\left\{\rho \in \Omega_{+}:|\rho| \geq \varepsilon,\left|\rho-\rho_{v}\right| \geq \varepsilon, v=\overline{1, n_{-}}\right\}$.

Let $\kappa_{v}, v \in \overline{1, n_{-}}$be the multiplicity of $\lambda_{v}$ as a zero of $\Delta(\lambda)$. Then for any $\varepsilon>0$ and $T>T_{\varepsilon}$ all the zeros of $\Delta^{(T)}(\lambda)$ are: $\lambda_{v j}^{(T)}=\left(\rho_{v j}^{(T)}\right)^{2}, j=\overline{1, \kappa_{v}}, v=\overline{1, n_{-}}, \lambda_{0 j}^{(T)}=\left(\rho_{0 j}^{(T)}\right)^{2}, j=\overline{1, \kappa_{0}^{(T)}}$, where $\left|\rho_{v j}^{(T)}-\rho_{v}\right|<\varepsilon,\left|\rho_{0 j}^{(T)}\right|<\varepsilon$. Although $\kappa_{0}^{(T)}$ depends on $T$ it follows from Lemma 2.3 that $\kappa_{0}^{(T)} \leq$ $N^{*}$, where $N^{*}$ does not depend on $T$. We set $\rho_{0 j}^{(T)}=0, j=\overline{\kappa_{0}^{(T)}+1, N^{*}}$.

Consider the family of functions

$$
f^{(T)}(\rho)=\gamma_{k k}^{(T)}(\rho) \cdot \prod_{v=1}^{n_{-}} \prod_{j=1}^{\kappa_{v}} \frac{\rho-\rho_{v j}^{(T)}}{\rho-\bar{\rho}_{v j}^{(T)}} \cdot \prod_{j=1}^{N^{*}} \frac{\rho-\rho_{0 j}^{(T)}}{\rho-\bar{\rho}_{0 j}^{(T)}} .
$$

It is clear that $\rho_{v j}^{(T)} \rightarrow \rho_{v}, \rho_{0 j}^{(T)} \rightarrow 0$ as $T \rightarrow \infty$. So for any fixed $\rho \in \Omega_{+} f^{(T)}(\rho) \rightarrow f(\rho)$, where:

$$
f(\rho)=\gamma_{k k}(\rho) \prod_{v=1}^{n_{-}}\left(\frac{\rho-\rho_{v}}{\rho-\bar{\rho}_{v}}\right)^{\kappa_{v}} .
$$

On the other hand Lemma 3.7 and Remark 3.4 yield the estimate $\left|f^{(T)}(\rho)\right| \leq A_{k}$, consequently $|f(\rho)| \leq A_{k}$. In particular, this means that $\gamma_{k k}(\rho)=O(1)$ as $\rho \rightarrow 0, \rho \in \Omega_{+}$. On the other hand the classical theory guarantees the $\delta_{k}(\rho)=O(1)$ as $\rho \rightarrow 0, \rho \in \Omega_{+}$and this completes the proof.

Now we agree that together with $L=L(q, G)$ we consider an operator $\tilde{L}=L(\tilde{q}, G)$ on the same graph $G$ but having a different potential $\tilde{q}(x)$. If a certain symbol $\xi$ denotes an object related to $L$, then the corresponding symbol $\tilde{\xi}$ with tilde denotes the analogous object related to $\tilde{L}$ and $\hat{\xi}:=\xi-\tilde{\xi}$.

Lemma 3.9. For $\rho \in \overline{\Omega_{+}^{\delta}}$ one gets:

$$
\psi_{k k}\left(x_{k}, \rho\right)=O\left(\exp \left(-i \rho x_{k}\right)\right), \psi_{k k}^{\prime}\left(x_{k}, \rho\right)=O\left(\rho \exp \left(-i \rho x_{k}\right)\right),
$$




$$
\hat{\psi}_{k k}\left(x_{k}, \rho\right)=O\left(\frac{1}{\rho} \exp \left(-i \rho x_{k}\right)\right)
$$

Proof. Using the representation (2.3) one can easily verify that

$$
\hat{\Delta}(\lambda)=O\left(\frac{1}{\rho} \exp (\tau \pi)\right) .
$$

In a similar way one can obtain:

$$
\hat{\Delta}_{k}(\lambda)=O\left(\frac{1}{\rho^{2}} \exp (\tau \pi)\right) .
$$

Using representation (3.2), estimates (3.10), (3.11), Lemma 2.8 and taking into account the classical estimates:

$$
\begin{aligned}
& e_{k}\left(x_{k}, \rho\right)=O\left(\mathrm{e}^{i \rho x_{k}}\right), \hat{e}_{k}\left(x_{k}, \rho\right)=O\left(\frac{1}{\rho} \mathrm{e}^{i \rho x_{k}}\right), \\
& S_{k}\left(x_{k}, \lambda\right)=O\left(\frac{1}{\rho} \mathrm{e}^{-i \rho x_{k}}\right), \hat{S}_{k}\left(x_{k}, \lambda\right)=O\left(\frac{1}{\rho^{2}} \mathrm{e}^{-i \rho x_{k}}\right),
\end{aligned}
$$

we obtain the required assertion.

Definition 3.1. The data $J_{k}:=\left\{s_{k}(\rho), \rho \in \mathbf{R} \backslash\left(\{0\} \cup Z_{0}^{+}\right), Z_{k}^{-}, \alpha_{k}(\rho), \rho \in Z_{k}^{-}\right\}$are called the scattering data, associated with $\mathscr{E}_{k}$.

Next theorem shows that the scattering data, associated with $\mathscr{E}_{k}$ uniquely determine the potential $q_{k}\left(x_{k}\right)$ on the edge.

Theorem 3.1. Let $L, \tilde{L}$ be Sturm-Liouville operators on $G$ with real-valued potentials $q(\cdot)$ and $\tilde{q}(\cdot)$ both satisfying (1.2). Then $J_{k}=\tilde{J}_{k}$ yields $q_{k}\left(x_{k}\right)=\tilde{q}_{k}\left(x_{k}\right)$.

Proof. Consider for $\lambda \in \mathbf{C} \backslash[0,+\infty)$ the following functions:

$$
\varphi_{1}\left(x_{k}, \lambda\right):=\psi_{k k}\left(x_{k}, \rho\right), \quad \varphi_{2}\left(x_{k}, \lambda\right):=e_{k}\left(x_{k}, \rho\right), \quad \lambda=\rho^{2}, \rho \in \Omega_{+} .
$$

Let us define the matrices

$$
\Psi\left(x_{k}, \lambda\right):=\left[\begin{array}{l}
\varphi_{1}\left(x_{k}, \lambda\right) \varphi_{2}\left(x_{k}, \lambda\right) \\
\varphi_{1}^{\prime}\left(x_{k}, \lambda\right) \varphi_{2}^{\prime}\left(x_{k}, \lambda\right)
\end{array}\right]
$$

and $\tilde{\Psi}\left(x_{k}, \lambda\right)$ and introduce the spectral mapping matrix:

$$
P\left(x_{k}, \lambda\right):=\Psi\left(x_{k}, \lambda\right) \tilde{\Psi}^{-1}\left(x_{k}, \lambda\right) .
$$

It follows from Lemma 3.5 that for the limit-value matrices $\Psi^{ \pm}\left(x_{k}, \lambda\right):=\Psi\left(x_{k}, \lambda \pm i 0\right)$, $\lambda \in(0,+\infty) \backslash \Lambda$ the following relation holds:

$$
\Psi^{-}\left(x_{k}, \lambda\right)=\Psi^{+}\left(x_{k}, \lambda\right) w(\lambda),
$$


where

$$
w(\lambda)=\left[\begin{array}{ll}
\overline{s_{k}(\rho)} & 1 \\
1-\left|s_{k}(\rho)\right|^{2}-s_{k}(\rho)
\end{array}\right], \quad \lambda=\rho^{2}, \rho \in(0,+\infty) .
$$

Suppose that $s_{k}=\tilde{s}_{k}$. Then $w=\tilde{w}$ and consequently $P^{+}\left(x_{k}, \lambda\right)=P^{-}\left(x_{k}, \lambda\right), \lambda \in(0,+\infty) \backslash$ $(\Lambda \cup \tilde{\Lambda})$. This means that $P\left(x_{k}, \lambda\right)$ is holomorphic in $\lambda \in \mathbf{C} \backslash(\{0\} \cup \Lambda \cup \tilde{\Lambda})$. Take an arbitrary $\lambda_{0} \in(0,+\infty) \cap(\Lambda \cup \tilde{\Lambda})$. It follows from Lemma 3.4 that $P\left(x_{k}, \lambda\right)$ is bounded in the neighborhood of $\lambda_{0}$, so $\lambda_{0}$ is a removable singularity for $P\left(x_{k}, \lambda\right)$.

Then, $J_{k}=\tilde{J}_{k}$ means in particular that $\Lambda^{-}=\tilde{\Lambda}^{-}$. Taking an arbitrary $\lambda_{0} \in \Lambda^{-}$we can conclude that $\lambda_{0}$ is either a pole or a removable singularity for $P\left(x_{k}, \lambda\right)$. Let us consider the functions $P_{11}\left(x_{k}, \lambda\right)$ and $P_{12}\left(x_{k}, \lambda\right)$. One has:

$$
\begin{aligned}
& P_{11}\left(x_{k}, \lambda\right)=\frac{1}{2 i \rho}\left(\psi_{k k}\left(x_{k}, \rho\right) \tilde{e}_{k}^{\prime}\left(x_{k}, \rho\right)-\tilde{\psi}_{k k}^{\prime}\left(x_{k}, \rho\right) e_{k}\left(x_{k}, \rho\right)\right), \\
& P_{12}\left(x_{k}, \lambda\right)=\frac{1}{2 i \rho}\left(\tilde{\psi}_{k k}\left(x_{k}, \rho\right) e_{k}\left(x_{k}, \rho\right)-\psi_{k k}\left(x_{k}, \rho\right) \tilde{e}_{k}\left(x_{k}, \rho\right)\right) .
\end{aligned}
$$

Substituting here the representations (see Remark 3.2)

$$
\begin{aligned}
& \psi_{k k}\left(x_{k}, \rho\right)=\frac{i \alpha_{k}\left(\rho_{0}\right)}{\rho-\rho_{0}} e_{k}\left(x_{k}, \rho_{0}\right)+O(1), \quad \rho \rightarrow \rho_{0}, \\
& \tilde{\psi}_{k k}\left(x_{k}, \rho\right)=\frac{i \tilde{\alpha}_{k}\left(\rho_{0}\right)}{\rho-\rho_{0}} \tilde{e}_{k}\left(x_{k}, \rho_{0}\right)+O(1), \quad \rho \rightarrow \rho_{0},
\end{aligned}
$$

and taking into account that $\alpha_{k}\left(\rho_{0}\right)=\tilde{\alpha}_{k}\left(\rho_{0}\right)$ we obtain $P_{11}\left(x_{k}, \lambda\right)=O(1), P_{12}\left(x_{k}, \lambda\right)=O(1)$ in a neighborhood of $\lambda_{0}$. Thus $\lambda_{0}$ is a removable singularity.

Then, using Lemma 3.9 and the classical asymptotics for the Jost solution $e_{k}\left(x_{k}, \rho\right)$ one can obtain the estimates:

$$
P_{11}\left(x_{k}, \lambda\right)-1=O\left(\frac{1}{\rho}\right), \quad P_{12}\left(x_{k}, \lambda\right)=O\left(\frac{1}{\rho}\right), \quad \lambda \rightarrow \infty, \rho^{2}=\lambda, \rho \in \overline{\Omega_{+}^{\delta}} .
$$

On the other hand Lemma 3.8 yields:

$$
P_{11}\left(x_{k}, \lambda\right)-1=O\left(\frac{1}{\rho}\right), \quad P_{12}\left(x_{k}, \lambda\right)=O\left(\frac{1}{\rho}\right), \quad \lambda \rightarrow 0, \rho^{2}=\lambda .
$$

These estimates together mean that actually $P_{11}\left(x_{k}, \lambda\right)-1=0, P_{12}\left(x_{k}, \lambda\right)=0$, i.e. $\varphi_{v}\left(x_{k}, \lambda\right)=$ $\tilde{\varphi}_{v}\left(x_{k}, \lambda\right), v=1,2$ and, consequently, $q_{k}\left(x_{k}\right)=\tilde{q}_{k}\left(x_{k}\right)$ for a.e. $x_{k} \in[0, \infty)$.

Thus we can formulate the following particular inverse scattering problem.

Problem $I P(k)$. Given the scattering data $J_{k}$, construct $q_{k}(\cdot)$.

Our next goal is to reduce the Problem $I P(k)$ to a certain linear equation in some Hilbert space. 
We start with the identities from Lemma 3.5 written for $L$ and $\tilde{L}$. After subtraction we obtain:

$$
\hat{\psi}_{k k}\left(x_{k}, \rho\right)=\hat{e}_{k}^{-}\left(x_{k}, \rho\right)+s_{k}(\rho) \hat{e}_{k}\left(x_{k}, \rho\right)+\hat{s}_{k}(\rho) \tilde{e}_{k}\left(x_{k}, \rho\right),
$$

where (and below) we denote $e_{k}^{-}\left(x_{k}, \rho\right):=e_{k}\left(x_{k},-\rho\right)$. Then we integrate this relation as follows:

$$
\begin{aligned}
& \frac{1}{2 \pi i} \int_{-N-1 / 2}^{N+1 / 2} \frac{\mathrm{e}^{i \mu x_{k}}}{\mu-\rho} \hat{\psi}_{k k}\left(x_{k}, \mu\right) d \mu=\frac{1}{2 \pi i} \int_{-N-1 / 2}^{N+1 / 2} \frac{\mathrm{e}^{i \mu x_{k}}}{\mu-\rho} \hat{e}_{k}^{-}\left(x_{k}, \mu\right) d \mu \\
& \quad+\frac{1}{2 \pi i} \int_{-N-1 / 2}^{N+1 / 2} \frac{\mathrm{e}^{i \mu x_{k}}}{\mu-\rho} s_{k}(\mu) \hat{e}_{k}\left(x_{k}, \mu\right) d \mu+\frac{1}{2 \pi i} \int_{-N-1 / 2}^{N+1 / 2} \frac{\mathrm{e}^{i \mu x_{k}}}{\mu-\rho} \hat{s}_{k}(\mu) \tilde{e}_{k}\left(x_{k}, \mu\right) d \mu,
\end{aligned}
$$

where $\rho \in \Omega_{-}=\{\rho: \operatorname{Im} \rho<0\}$ is arbitrary fixed, $N$ is positive integer. Our next step is taking the limit as $N \rightarrow \infty$. It follows from Lemma 3.9 that:

$$
\begin{aligned}
& \lim _{N \rightarrow \infty} \frac{1}{2 \pi i} \int_{-N-1 / 2}^{N+1 / 2} \frac{\mathrm{e}^{i \mu x_{k}}}{\mu-\rho} \hat{\psi}_{k k}\left(x_{k}, \mu\right) d \mu=\lim _{N \rightarrow \infty} \frac{1}{2 \pi i} \int_{\Gamma_{N}^{+}} \frac{\mathrm{e}^{i \mu x_{k}}}{\mu-\rho} \hat{\psi}_{k k}\left(x_{k}, \mu\right) d \mu \\
& \quad=\sum_{\mu \in Z_{k}^{-}} i \alpha_{k}(\mu) \frac{\mathrm{e}^{i \mu x_{k}}}{\mu-\rho} e_{k}\left(x_{k}, \mu\right),
\end{aligned}
$$

where $\Gamma_{N}^{+}=[-N-1 / 2, N+1 / 2] \cup\{(N+1 / 2) \exp (i \beta), \beta \in(0, \pi)\}$ with the counterclockwise orientation. Analogously one gets:

$$
\begin{aligned}
\lim _{N \rightarrow \infty} & \frac{1}{2 \pi i} \int_{-N-1 / 2}^{N+1 / 2} \frac{\mathrm{e}^{i \mu x_{k}}}{\mu-\rho} \hat{e}_{k}^{-}\left(x_{k}, \mu\right) d \mu \\
\quad & =\lim _{N \rightarrow \infty} \frac{1}{2 \pi i} \int_{\Gamma_{N}^{-}} \frac{\mathrm{e}^{i \mu x_{k}}}{\mu-\rho} \hat{e}_{k}^{-}\left(x_{k}, \mu\right) d \mu=-\hat{e}_{k}^{-}\left(x_{k}, \rho\right) \mathrm{e}^{i \rho x_{k}},
\end{aligned}
$$

where $\Gamma_{N}^{+}=[-N-1 / 2, N+1 / 2] \cup\{(N+1 / 2) \exp (i \beta), \beta \in(-\pi, 0)\}$ with the clockwise orientation. Further, the estimates $\left|s_{k}(\rho)\right|<1, \hat{e}_{k}\left(x_{k}, \rho\right)=O\left(\rho^{-1}\right)$ show that

$$
\begin{gathered}
\lim _{N \rightarrow \infty} \frac{1}{2 \pi i} \int_{-N-1 / 2}^{N+1 / 2} \frac{\mathrm{e}^{i \mu x_{k}}}{\mu-\rho} s_{k}(\mu) \hat{e}_{k}\left(x_{k}, \mu\right) d \mu \\
=\frac{1}{2 \pi i} \int_{-\infty}^{\infty} \frac{\mathrm{e}^{i \mu x_{k}}}{\mu-\rho} s_{k}(\mu) \hat{e}_{k}\left(x_{k}, \mu\right) d \mu
\end{gathered}
$$

and integral in right-hand side converges absolutely. It follows from (3.12)-(3.15) that there exists a limit:

$$
\lim _{N \rightarrow \infty} \frac{1}{2 \pi i} \int_{-N-1 / 2}^{N+1 / 2} \frac{\mathrm{e}^{i \mu x_{k}}}{\mu-\rho} \hat{s}_{k}(\mu) \tilde{e}_{k}\left(x_{k}, \mu\right) d \mu=: G_{k}\left(x_{k}, \rho\right) .
$$

In view of (3.13)-(3.16) the relation (3.12) can be rewritten in the following form:

$$
\hat{e}_{k}^{-}\left(x_{k}, \rho\right)=\sum_{\mu \in Z_{k}^{-}} i \alpha_{k}(\mu) \frac{\mathrm{e}^{i(\mu-\rho) x_{k}}}{\rho-\mu} \hat{e}_{k}\left(x_{k}, \mu\right)+\frac{1}{2 \pi i} \int_{-\infty}^{\infty} \frac{\mathrm{e}^{i(\mu-\rho) x_{k}}}{\mu-\rho} s_{k}(\mu) \hat{e}_{k}\left(x_{k}, \mu\right) d \mu
$$




$$
+G_{k}\left(x_{k}, \rho\right) \mathrm{e}^{-i \rho x_{k}}+\sum_{\mu \in Z_{k}^{-}} i \alpha_{k}(\mu) \frac{\mathrm{e}^{i(\mu-\rho) x_{k}}}{\rho-\mu} \tilde{e}_{k}\left(x_{k}, \mu\right), \quad \rho \in \Omega_{-} .
$$

While solving the Problem $I P(k)$ we assume the operator $\tilde{L}$ to be known ("model") and consequently we can assume all the values in (3.17) except $\hat{e}_{k}\left(x_{k}, \rho\right)$ to be given. Thus, our goal is to obtain from (3.17) some closed system of equations with respect to the values of $\hat{e}_{k}\left(x_{k}, \rho\right)$. For this purpose we set in (3.17) $\rho \in-Z_{k}^{-}$and then (independently) take the limit as $\operatorname{Im} \rho \rightarrow-0$. The resulted pair of relations we write as a certain equation in some Hilbert space.

Let us define:

$$
\begin{aligned}
& g_{k}\left(x_{k}, \rho\right):=G_{k}\left(x_{k}, \rho\right) \mathrm{e}^{-i \rho x_{k}}+\sum_{\mu \in Z_{k}^{-}} i \alpha_{k}(\mu) \frac{\mathrm{e}^{i(\mu-\rho) x_{k}}}{\rho-\mu} \tilde{e}_{k}\left(x_{k}, \mu\right), \quad \rho \in-Z_{k}^{-}, \\
& g_{k}\left(x_{k}, \rho\right):=\lim _{\varepsilon \rightarrow+0} G_{k}\left(x_{k}, \rho-i \varepsilon\right) \mathrm{e}^{-i(\rho-i \varepsilon) x_{k}}+\sum_{\mu \in Z_{k}^{-}} i \alpha_{k}(\mu) \frac{\mathrm{e}^{i(\mu-\rho) x_{k}}}{\rho-\mu} \tilde{e}_{k}\left(x_{k}, \mu\right), \quad \rho \in \mathbf{R} .
\end{aligned}
$$

The limit in (3.19) exists because it exists for all the other terms in (3.17).

Denote by $C$ the Cauchy operator:

$$
C f(\rho):=\frac{1}{2 \pi i} \int_{-\infty}^{\infty} \frac{f(\mu) d \mu}{\mu-\rho}, \quad \rho \in \mathbf{C} \backslash \mathbf{R}
$$

and define in $L_{2}(\mathbf{R})$ the operators:

$$
C^{ \pm} f(\rho):=\lim _{\varepsilon \rightarrow+0}(C f)(\rho \pm i \varepsilon) .
$$

Also we shall use the following operators acting (and continuous) in $L_{2}(\mathbf{R})$ :

$$
U_{k} f(\rho):=s_{k}(\rho) f(\rho), V_{k}\left(x_{k}\right) f(\rho):=\mathrm{e}^{i \rho x_{k}} f(\rho), T f(\rho):=f(-\rho) .
$$

Let $\mathscr{H}$ be a space of functions $f(\rho), \rho \in \mathbf{R} \cup\left(-Z_{k}^{-}\right)$such that $\left.f\right|_{\mathbf{R}} \in L_{2}(\mathbf{R})$. We consider $\mathscr{H}$ as a Hilbert space with the scalar product of the form:

$$
\left(f_{1}, f_{2}\right):=\frac{1}{2 \pi} \int_{-\infty}^{\infty} f_{1}(\rho) \overline{f_{2}(\rho)} d \rho+\sum_{\rho \in-Z_{k}^{-}} \alpha_{k}(-\rho) f_{1}(\rho) \overline{f_{2}(\rho)} .
$$

Let us define in $\mathscr{H}$ the following operators depending on parameter $x_{k}$ :

$$
\begin{aligned}
& H_{k}\left(x_{k}\right) f(\rho):=V_{k}^{-1}\left(x_{k}\right) C^{-} U_{k} V_{k}\left(x_{k}\right) T f(\rho)+\sum_{\mu \in Z_{k}^{-}} i \alpha_{k}(\mu) \frac{\mathrm{e}^{i(\mu-\rho) x_{k}}}{\rho-\mu} f(-\mu), \quad \rho \in \mathbf{R} \\
& H_{k}\left(x_{k}\right) f(\rho):=\frac{1}{2 \pi i} \int_{\infty}^{\infty} s_{k}(\mu) \frac{\mathrm{e}^{i(\mu-\rho) x_{k}}}{\mu-\rho} f(-\mu) d \mu+\sum_{\mu \in Z_{k}^{-}} i \alpha_{k}(\mu) \frac{\mathrm{e}^{i(\mu-\rho) x_{k}}}{\rho-\mu} f(-\mu), \quad \rho \in Z_{k}^{-} .
\end{aligned}
$$

Thus, from (3.17) we arrive at the following relation:

$$
\hat{e}_{k}^{-}\left(x_{k}\right)=H_{k}\left(x_{k}\right) \hat{e}_{k}^{-}\left(x_{k}\right)+g_{k}\left(x_{k}\right)
$$


where $x_{k}$ is considered as a parameter, $\hat{e}_{k}^{-}\left(x_{k}\right)$ denotes the function $\hat{e}_{k}^{-}\left(x_{k}, \cdot\right)$ which is considered as an element of $\mathscr{H}$. Since $H_{k}\left(x_{k}\right)$ is continuous in $\mathscr{H}$ the function $g_{k}\left(x_{k}, \rho\right), \rho \in$ $\mathbf{R} \cup\left(-Z_{k}^{-}\right)$belongs to $\mathscr{H}$, we denote by $g_{k}\left(x_{k}\right)$ the corresponding element of this space.

Theorem 3.2. For each fixed $x_{k} \in[0, \infty) \hat{e}_{k}^{-}\left(x_{k}\right)$ is a unique solution of the equation (3.25) in the space $\not{H}$.

Proof. Theorem is already proved in part by the considerations above. Now we are to show that $\hat{e}_{k}^{-}\left(x_{k}\right)$ is a unique solution of (3.25). For this purpose we consider the corresponding homogenous equation:

$$
f=H_{k}\left(x_{k}\right) f
$$

for arbitrary fixed $x_{k}$. First, using the symmetries $s_{k}(-\rho)=\overline{s_{k}(\rho)}, \rho \in \mathbf{R}, \alpha_{k}(\rho)=\overline{\alpha_{k}(\rho)}, \rho \in Z_{k}^{-}$ one can easily obtain the following fact: if $f$ is a solution of (3.26) then $\varphi$, defined as follows:

$$
\varphi(\rho):=i(\overline{f(\rho)}-f(-\rho)), \rho \in \mathbf{R}, \quad \varphi(\rho):=i(\overline{f(\rho)}-f(\rho)), \rho \in-Z_{k}^{-}
$$

satisfies (3.26) as well.

Now let $\varphi \in \mathscr{H}$ be a solution of (3.26). Without loss of generality we can assume the following properties:

$$
\varphi(-\rho)=\overline{\varphi(\rho)}, \rho \in \mathbf{R}, \quad \varphi(\rho)=\overline{\varphi(\rho)}, \rho \in-Z_{k}^{-} .
$$

Then one gets:

$$
(\varphi, \varphi)=\left(H_{k}\left(x_{k}\right) \varphi, \varphi\right)=A_{11}+A_{22}+A_{12}+A_{21}
$$

where:

$$
\begin{aligned}
& A_{11}=\frac{1}{2 \pi}\left(\left.\left(H_{k}\left(x_{k}\right) \varphi\right)\right|_{\mathbf{R}},\left.\varphi\right|_{\mathbf{R}}\right)_{L_{2}(\mathbf{R})}, \\
& A_{22}=\sum_{\rho \in-Z_{k}^{-}} \alpha_{k}(-\rho) \overline{\varphi(\rho)} \sum_{\mu \in Z_{k}^{-}} i \alpha_{k}(\mu) \varphi(-\mu) \frac{\mathrm{e}^{i(\mu-\rho) x_{k}}}{\rho-\mu}, \\
& A_{12}=\frac{1}{2 \pi} \int_{\mathbf{R}} \sum_{\mu \in Z_{k}^{-}} i \alpha_{k}(\mu) \frac{\mathrm{e}^{i(\mu-\rho) x_{k}}}{\rho-\mu} \varphi(-\mu) \overline{\varphi(\rho)} d \rho, \\
& A_{21}=\frac{1}{2 \pi i} \int_{\mathbf{R}} \sum_{\rho \in-Z_{k}^{-}} \alpha_{k}(-\rho) s_{k}(\mu) \frac{\mathrm{e}^{i(\mu-\rho) x_{k}}}{\mu-\rho} \varphi(-\mu) \overline{\varphi(\rho)} d \mu .
\end{aligned}
$$

First, using (3.27) and the formula:

$$
\frac{\mathrm{e}^{i(\mu-\rho) x_{k}}}{i(\mu-\rho)}=-\int_{x_{k}}^{\infty} \mathrm{e}^{i(\mu-\rho) t} d t, \quad \operatorname{Im} \mu>\operatorname{Im} \rho,
$$


we transform $A_{22}$ into the form:

$$
A_{22}=-\int_{x_{k}}^{\infty} \Phi^{2}(t) d t, \quad \Phi(t)=\sum_{\rho \in-Z_{k}^{-}} \alpha_{k}(-\rho) \varphi(\rho) \mathrm{e}^{-i \rho t} .
$$

Proceeding in analogous way we obtain after some algebra:

$$
A_{12}+A_{21}=-\int_{x_{k}}^{\infty} \Phi(t) \Psi(t) d t
$$

where $\Psi(t)$ is the Fourier transform:

$$
\Psi(t)=\frac{1}{2 \pi} \text { l.i.m.N } \int_{-N}^{N} f(\rho) \mathrm{e}^{i \rho t} d \rho, \quad f(\rho):=\varphi(\rho)+s_{k}(\rho) \varphi(-\rho) .
$$

In order to calculate $\Psi(t)$ we have to return to (3.26) for $\rho \in \mathbf{R}$. Using the relation $C^{+}-C^{-}=E$ (identical operator) we obtain:

$$
\varphi(\rho)+s_{k}(\rho) \varphi(-\rho)=\sum_{\mu \in Z_{k}^{-}} i \alpha_{k}(\mu) \frac{\mathrm{e}^{i(\mu-\rho) x_{k}}}{\rho-\mu} \varphi(-\mu)+V_{k}^{-1}\left(x_{k}\right) C^{+} U_{k} V_{k}\left(x_{k}\right) T \varphi(\rho) .
$$

Since the Fourier transform of the last term in right-hand side is equal to 0 for all $t>x_{k}$ we deduce from this relation:

$$
\Psi(t)=-\sum_{\mu \in Z_{k}^{-}} \alpha_{k}(\mu) \varphi(-\mu) \mathrm{e}^{i \mu t}=-\Phi(t)
$$

Substituting this into (3.29), (3.30) we obtain $A_{12}+A_{21}+A_{22}=0$.

It follows from (3.27), the symmetry $s_{k}(-\rho)=\overline{s_{k}(\rho)}$ and the estimate $\left|s_{k}(\rho)\right|<1$ that if $\varphi \neq 0$ then

$$
\left|A_{11}\right|=\frac{1}{2 \pi}\left|\left(\left.\left(H_{k}\left(x_{k}\right) \varphi\right)\right|_{\mathbf{R}},\left.\varphi\right|_{\mathbf{R}}\right)_{L_{2}(\mathbf{R})}\right|<\frac{1}{2 \pi}\left(\left.\varphi\right|_{\mathbf{R}},\left.\varphi\right|_{\mathbf{R}}\right)_{L_{2}(\mathbf{R})} \leq(\varphi, \varphi) .
$$

On the other hand as we have already seen above $\varphi=H_{k}\left(x_{k}\right) \varphi$ implies $(\varphi, \varphi)=\left(H_{k}\left(x_{k}\right) \varphi, \varphi\right)=$ $A_{11}$. Thus, we conclude that $\varphi=0$ and this completes the proof.

Theorem 3.2 provides the following procedure for solving the Problem $I P(k)$.

Algorithm 3.1. Given the scattering data $J_{k}$.

1. Choose an arbitrary "model" operator with a real-valued potential $\tilde{q}$ satisfying (1.2). For instance, one can assume $\tilde{q}_{j}=0, j=\overline{0, p}$.

2. For each fixed $x_{k} \in[0, \infty)$ construct the operator $H_{k}\left(x_{k}\right)$ via (3.20), (3.21), (3.23), (3.24) and calculate $g_{k}\left(x_{k}\right)$ by (3.18), (3.19).

3. For each fixed $x_{k} \in[0, \infty)$ find $\hat{e}_{k}^{-}\left(x_{k}\right)$ by solving the equation (3.25). 
4. Take an arbitrary $\rho \in \mathbf{R} \backslash\{0\}$. For each fixed $x_{k} \in[0, \infty)$ calculate $e_{k}^{-}\left(x_{k}, \rho\right)=\tilde{e}_{k}^{-}\left(x_{k}, \rho\right)+$ $\hat{e}_{k}^{-}\left(x_{k}, \rho\right)$.

5. Recover $q_{k}\left(x_{k}\right)=\left(e_{k}^{-}\left(x_{k}, \rho\right)\right)^{\prime \prime} / e_{k}^{-}\left(x_{k}, \rho\right)+\rho^{2}$.

\section{Inverse scattering problem}

It turns out that in order to recover the operator on the entire graph $G$ one should have all the particular scattering data $J_{k}, k=\overline{1, p}$, described above and some additional information.

Let $\Lambda^{0}$ and $\Lambda_{0}$ be the spectra of periodic and Dirichlet-Dirichlet boundary-value problems on $\mathscr{E}_{0}$ respectively. We define the function $\omega(\lambda), \lambda \in \Lambda^{-}$setting $\omega(\lambda)=1$ if $\lambda \in \Lambda^{0} \cap \Lambda_{0}$ and $\omega(\lambda)=0$ otherwise. Suppose now that all the eigenvalues of periodic problem are: $\lambda_{0}^{0}<$ $\lambda_{3}^{0} \leq \lambda_{4}^{0}<\lambda_{7}^{0} \leq \lambda_{8}^{0}<\ldots$ (here we use the classical notations which take into account the multiplicities of eigenvalues). Let $\lambda_{1}^{0} \leq \lambda_{2}^{0}<\lambda_{5}^{0} \leq \lambda_{6}^{0}<\ldots$ be the eigenvalues for the antiperiodic problem and $\sigma_{n}^{0}, n=\overline{1, \infty}$ be the sign sequence corresponding to the spectrum $\Lambda_{0}=\left\{\mu_{n}\right\}_{n=1}^{\infty}$ of Dirichlet-Dirichlet problem, where we set $\sigma_{n}^{0}=0$ if $\lambda_{2 n-1}^{0}=\lambda_{2 n}^{0}$.

Definition 4.1. The data

$$
J=\left\{J_{k}, k=\overline{1, p} ; \Lambda ; \omega(\lambda), \lambda \in \Lambda^{-} ; \sigma_{n}^{0}, n=\overline{1, \infty}\right\}
$$

are called the global scattering data.

Now we can formulate the inverse scattering problem on the whole graph $G$.

Problem $I P$. Given the global scattering data $J$, construct $q_{k}(\cdot), k=\overline{0, p}$.

The procedure described in the previous section allows us to reconstruct the functions $q_{k}(\cdot), k=\overline{1, p}$ from the given scattering data. The final step is reconstruction of $q_{0}\left(x_{0}\right), x_{0} \in$ $[0, \pi]$. Now we show how to reduce this problem to the classical inverse periodic problem [20] (when all other $q_{k}(\cdot), k=\overline{1, p}$ have been already recovered as we mentioned above).

First we note that we can consider the Jost solutions $e_{j}\left(x_{j}, \rho\right), j=\overline{1, p}$ as known. In particular we can calculate the local Weyl functions:

$$
m_{j}(\lambda)=\frac{e_{j}^{\prime}(0, \rho)}{e_{j}(0, \rho)}, \rho \in \bar{\Omega}_{+}, j=\overline{1, p}
$$

Also we can calculate the values $\psi_{k k}(0, \rho), \psi_{k k}^{\prime}(0, \rho), \rho \in \bar{\Omega}_{+}$, where $k \in \overline{1, p}$ is arbitrary fixed. For this purpose one can use the relation from Lemma 3.5 for real $\rho$ and then make an analytic continuation. Now we can write the matching condition (1.3) for $\psi_{k}(x, \rho)$ in the following form:

$$
m_{0}(\lambda)=-\frac{\psi_{k k}^{\prime}(0, \rho)}{\psi_{k k}(0, \rho)}-\sum_{j=\frac{\sum_{1, p} \backslash\{k\}}{}} m_{j}(\lambda), \quad \lambda=\rho^{2}, \rho \in \Omega_{+} .
$$


This relation provides a way to find $m_{0}(\cdot)$ which is the fraction of the form specified in section 2. Let $Z_{0}$ be a set of poles of $m_{0}(\cdot)$. It is clear that (see also proof of Lemma 2.7) $\Lambda_{0}=Z_{0} \cup \Lambda^{+} \cup$ $\left\{\lambda \in \Lambda^{-}: \omega(\lambda)=1\right\}$. So we can recover $\Lambda_{0}$ and corresponding characteristic function $d_{0}(\cdot)$ of the Dirichlet-Dirichlet problem using the formula [20]:

$$
d_{0}(\lambda)=\pi \prod_{n=1}^{\infty} \frac{\mu_{n}-\lambda}{n^{2}}
$$

Now we can return to $m_{0}(\cdot)$ and use $d_{0}(\cdot)$ to recover $d^{0}(\cdot)$. Given $\Lambda_{0}, \Lambda^{0}$ and a sign sequence $\left\{\right.$ sig $\left.m a_{n}^{0}\right\}$ we are able to pose the classical periodic inverse problem [20]. Thus we arrive at the following procedure.

Algorithm 4.1. Given the global scattering data $J$.

1. For each $k=\overline{1, p}$ construct $q_{k}(\cdot)$, by solving the Problem $I P(k)$ according to Algorithm 3.1.

2. Calculate $e_{k}\left(x_{k}, \rho\right), x_{k} \in[0, \infty), \rho \in \bar{\Omega}_{+}, k=\overline{1, p}$.

3. Take and fix an arbitrary $k \in \overline{1, p}$.

4. Construct $\psi_{k k}(0, \rho), \psi_{k k}^{\prime}(0, \rho)$ via Lemma 3.5 and then using a procedure of analytic continuation.

5. Calculate $m_{0}(\cdot)$ via $(4.1),(4.2)$.

6. Find the set $Z_{0}$ of poles of $m_{0}(\cdot)$ and construct the set $\Lambda_{0}=Z_{0} \cup \Lambda^{+} \cup\left\{\lambda \in \Lambda^{-}: \omega(\lambda)=1\right\}$ $=:\left\{\mu_{n}\right\}_{n=1}^{\infty}$.

7. Calculate $d_{0}(\cdot)$ using (4.3), $d^{0}(\cdot)$ as $d^{0}(\lambda)=m_{0}(\lambda) d_{0}(\lambda)$.

8. Given $d_{0}(\cdot), d^{0}(\cdot)$ and the sign sequence $\left\{\operatorname{sig} m a_{n}^{0}\right\}_{n=1}^{\infty}$ recover $q_{0}(\cdot)$, by solving the classical periodic inverse problem [20].

Theorem 4.1. Operator $L$ is uniquely determined by it's global scattering data and can be recovered from this data by using Algorithm 4.1.

\section{Acknowledgement}

This research was supported by the Russian Fund of Basic Research and the Taiwan National Science Council (projects 10-01-00099 and 10-01-92001-NSC-a).

\section{References}

[1] L. Faddeev and B. Pavlov, Model of free electrons and the scattering problem, Teor. Mat. Fiz., 55(1983), 257-269 (Russian); English transl. in Theor. Math. Phys., 55 (1983), 485-492.

[2] S. Nicaise, Some results on spectral theory over networks, applied to nerve impulse transmission, Lect. Notes Math., 1771(1985), 532-541. 
[3] P. Exner, Contact interactions on graph superlattices, J . Phys. A: Math. Gen., 29 (1996), 87-102.

[4] T. Kottos and U. Smilansky, Quantum chaos on graphs, Phys. Rev. Lett., 79 (1997), 4794-4797.

[5] Yu. V. Pokornyi and A. V. Borovskikh, Differential equations on networks (geometric graphs), J. Math. Sci. (N.Y.) 119(2004), 691-718.

[6] Yu. V. Pokornyi and V. Pryadiev, The qualitive Sturm-Liouville theory on spatial networks, J. Math. Sci. (N.Y.), 119, (2004), 788-835.

[7] M. I. Belishev, Boundary spectral inverse problem on a class of graphs (trees) by the BC method, Inverse Problems, 20 (2004), 647-672.

[8] B. M. Brown and R. Weikard, A Borg-Levinson theorem for trees, Proc. R. Soc. Lond. Ser. A Math. Phys. Eng. Sci., 461, (2005), 3231-3243.

[9] V. A. Yurko, Inverse spectral problems for Sturm-Liouville operators on graphs, Inverse Problems, 21 (2005), 1075-1086.

[10] V. A. Yurko, Recovering differential pencils on compact graphs, J. Diff. Equations, 244 (2008), 431-443.

[11] V. A. Yurko, Inverse problems for arbitrary order differential operators on trees, Matemat. Zametki, 83, (2008), 139-152 (Russian); English transl. in Math. Notes, 83(2008), 125-137.

[12] Yurko V.A., Uniqueness of recovering of Sturm-Liouville operators on A-graphs from spectra. Results in Mathematics 55, no.1-2 (2009), 199-207.

[13] V. A. Yurko, Inverse spectral problem for differential operators on arbitrary compact graphs, Journal of Inverse and Ill-Posed Problems, 18(2010).

[14] N.I. Gerasimenko, Inverse scattering problems on a noncompact graph, Teoret. Mat. Fiz., 74 (1988), 187-200; English transl. in Theor. Math. Phys., 75 (1988), 460-470.

[15] P. Kurasov and F. Sternberg, Om the inverse scattering problem on branching graphs, J. Phys. A, 35(2002), $101-121$.

[16] G. Freiling and V. A. Yurko, Inverse spectral problems for Sturm-Liouville operators on noncompact trees, Results in Math., 50 (2007), 195-212.

[17] I. Trooshin, V. Marchenko and K. Mochizuki, Inverse scattering on a graph containing circle, Analytic methods of analysis and DEs: AMADE 2006, 237-243, Camb. Sci. Publ., Cambridge, 2008.

[18] E. A. Coddington and N. Levinson, Theory of Ordinary Differential Equations, Mc Graw-Hill, New York, 1955.

[19] A. Zettl, Sturm-Liouville theory, Mathematical Surveys and Monographs 121. Providence, RI: American Mathematical Society, 2005.

[20] B. M. Levitan, Inverse Sturm-Liouville Problems, VNU Sci. Press, Utrecht, 1987.

Department of Mathematics, Saratov University, Astrakhanskaya 83, Saratov 410012, Russia.

E-mail: ignatievmu@info.sgu.ru 